\title{
Astrid Ackermann
}

\section{Tagungsprotokoll}

\section{Sektion: Binnensichten}

\section{Alfred Kobler: Österreich und die deutsche Nation-politische und kulturelle Distanz?}

Die Diskussion konzentriert sich auf das Verhältnis zwischen Österreich und der deutschen Nation in der Frühen Neuzeit sowie auf den Prozeß österreichischer Eigen- und Selbstbewußtwerdung. Das Kaisertum habe dabei als interne Klammer und Unterpfand der Zusammengehörigkeit mit der deutschen Geschichte fungiert. Die kulturelle Differenz zum restlichen Deutschland begründe sich dennoch vor allem aus der Politik des Kaisers. Beispielsweise sei die kaiserliche Besatzung Bayerns im Spanischen Erbfolgekrieg schon wenig später als österreichische wahrgenommen worden und habe einen breiten antiösterreichischen Diskurs provoziert. Anstöße von außen seien überhaupt für die Entwicklung eines österreichischen Sonderbewußtseins wiederholt maßgeblich gewesen: Dies gelte für die oft als Katalysator eines deutschen Nationalbewußtseins verstandene Türkengefahr, die aber auch das österreichische Sonderbewußtsein befördert und etwa die Apotheose des Kaiserhauses und des Prinzen Eugen ermöglicht habe. Ohne den preußischen Angriff hätte es die Staatsreformen Maria Theresias nicht gegeben. Selbst Philipp Wilhelm von Hörnigks Abhandlung „Österreich über alles, wann es nur will“ (1684) lasse sich als Argument für den österreichischen Gesamtstaat und dessen Leistungsfähigkeit, aber auch als Indiz für die Ähnlichkeit mit der deutschen Reichsnation lesen.

Es stelle sich daher die Frage, inwieweit die österreichische Entwicklung lediglich aus der Perspektive der heutigen Staatsverhältnisse beurteilt werde. Lasse sich ein ähnliches Bild der Entwicklung etwa für Hessen-Kassel zeichnen, wenn dieses Land ein selbständiger Staat geworden wäre? Auch andernorts fänden sich konstitutive Elemente wie in Österreich: konfessionelle Einheitlichkeit, eine prägende Dynastie (z.B. die Albertiner) und Klosterlandschaften (wie im schwäbisch-bayerischen Raum). Spezifisch für Österreich sei daher letztlich nur die Kaiserwürde, die den Habsburgern besondere Möglichkeiten geboten habe. Österreich sei daher sowohl als Staatsnation als auch als „Verwandtschaftsnation“ zu begreifen, denn das Haus Habsburg habe das Konnubium des Adels seiner Länder bewußt 
gefördert. Die sog. Hofratsnation (Ernst Bruckmüller), die in Alphons Lhotzkys Rede vom „österreichischen Menschen“ ihre Idealisierung erfahren habe, und damit die Beamten spielten ebenfalls eine wichtige Rolle für die Ausprägung des österreichischen Eigenbewußtseins.

\section{Peter Wilson: The Reichsarmee: Integration through Military Cooperation?}

Diskutiert werden vor allem Formen der normativen Integration durch das Reichskriegswesen und die Frage der Identität der Reichstruppen. Kollektive Identität setze aber eine spezifische Erinnerungskultur voraus wie der Fall „Roßbach" zeige. Hier bestehe eine gravierende Diskrepanz zwischen dem Geschehen und dessen Deutung. Das von Preußen lancierte Bild habe sich durchgesetzt: Obwohl am 5. November 1757 eine mit Reichstruppen lediglich verstärkte französische Armee besiegt worden sei, habe die preußische Publizistik einen Sieg über den französischen „Erbfeind“ und die „Reißausarmee“ gefeiert. Napoleon habe wohl deswegen die auf Veranlassung König Friedrichs II. bei Roßbach aufgestellte Siegessäule mit nach Paris nehmen lassen. Notwendig bleibe die Dekonstruktion der preußischen Legende. Dabei sei auch auf die militärischen Leistungen der Reichsarmee im weiteren Verlauf des Siebenjährigen Krieges zu verweisen, vor allem aber auf ihre politische Bedeutung und ihre symbolische Funktion für den Zusammenhalt von Reichsständen und Kaiser.

Hinsichtlich der identifikatorischen Kraft des Militärischen seien verschiedene Ebenen zu unterscheiden: die Reichsarmee in offiziellen Reichskriegen, die armierten Reichsstände als „Krisenfeuerwehr" für die Reichsverteidigung und schließlich die Kreisassoziationen. Im Falle der Reichsarmee sei zwischen einem möglichen nationalen Bewußtsein und der individuellen Identität der Soldaten zu differenzieren. Schwer einzuschätzen bleibe die Bedeutung der Konfession, wie das Beispiel der schwäbischen Kreistruppen zeige, die laut Befehl nicht über Religion hätten reden dürfen. Während die preußischen Regimenter über eigene Erinnerungskulturen verfügt hätten, sei bei der Reichs- als einer Kontingentarmee nichts Vergleichbares zu finden. Im Kontext des Prager Friedens zeige sich aber, daß ein gemeinsames deutsches „Vaterland“ erfahrbar gewesen sei, denn bei den Deutschen in schwedischen Diensten hätten die Flugschriften mit dem Appell, nicht gegen das eigene Vaterland Krieg zu führen, offenbar zu Gewissenskonflikten geführt.

\section{Alexander Schmidt: Debatten um die Vaterlandsliebe der Deutschen}

Die Diskussion steht ganz im Zeichen der Begriffe „Vaterland“ und „Vaterlandsliebe“. Festgestellt wird, daß sich „Vaterland“ bereits um 1500 in Reichsabschieden, Gelehrtenprotokollen und als rhetorisch verwandtes Bildungsgut findet. $\mathrm{Ob}$ dies handlungsleitend gewesen sei, ob tatsächlich von einem „Erfahrungsraum Deutschland" gesprochen werden könne, und ob nicht begrifflich zwischen der „patria“ und einem politisch aufladbaren „Patriotismus“ zu unterscheiden sei, 
wird intensiv debattiert. Schon im 16. Jahrhundert habe es die Selbstetikettierung als „Patriot“ gegeben, und der Begriff sei bereits polemisch und zur Parteienbildung gebraucht worden.

Das patriotische Vokabular werde offensichtlich in Krisenzeiten aktiviert, zunächst vor allem auf protestantischer, später auch auf katholischer Seite. Um 1800 erfolge eine begriffliche Übernahme der älteren Diskussion. Neu seien aber eine wesentlich breitere Trägerschicht, die Formulierung von Partizipationsvorstellungen, eine neue Medienlandschaft und die Reflexionsleistungen der Aufklärung. Wie das Beispiel Thomas Abbts zeige, gehe es nun nicht zuletzt um die Neupositionierung des Staates sowie um das Spannungsgefüge zwischen Weltbürgertum und Patriotismus. Die Verbindung des Einzelnen zum Ganzen durch religiös aufgeladene Begriffe werde von den Vertretern des Patriotismusdiskurses unterschiedlich bewertet: Abbt lehne anders als Melanchthon die Verknüpfung von Christ- und Patriotsein ab.

\section{Dieter Langewiesche: Föderative Nation, kulturelle Identität und politische Ordnung - (Rück-)Blick aus dem 19. Jabrbundert}

Die Darstellung der inneren Nationsbildung vor dem Nationalstaat, die Kritik am modernen Nationalstaat als Fluchtpunkt der Geschichtserzählung und die Betonung der föderalistischen Elemente in der deutschen Geschichte finden große Zustimmung. Der Föderalismus sei als durchgängige Kategorie der deutschen Geschichte auf zwei Ebenen - der politischen und derjenigen der Selbstorganisation der Bevölkerung - von der Frühen Neuzeit bis über 1871 hinaus zu verstehen. Das Alte Reich sei jedoch weder Vorläufer noch gar historisches Vorbild für die Europäische Union, die einen Bruch mit der europäischen Staatstradition darstelle.

Die Idee der föderativen Nation werde auch in den einzelstaatlichen Identifikationsangeboten mitgedacht. Beide stünden nicht in Widerspruch zueinander. Die Sänger, Schützen und Turner seien zunächst lokal, dann einzelstaatlich organisiert gewesen; gesamtdeutsche Treffen habe es vor allem in den 1840er Jahren gegeben, bei denen die Beteiligten mit Fahnen etc. die politisch-territoriale Vielgestaltigkeit dokumentiert hätten. Im Sinne von Karl W. Deutsch sei die Nationalbewegung als eine Nationalstaatsbewegung immer auch unter Einbeziehung der sozialen Aspekte zu verstehen.

Diskutiert wird die Bedeutung der Paulskirche und die Frage, ob dort in der Debatte um das Großherzogtum Posen ein „Radikalnationalismus“ feststellbar gewesen sei. Tatsächlich habe die Versammlung verschiedene Ansprüche begründet, aber prinzipiell - mit der Ausnahme gegenüber Polen - eine europaverträgliche kleindeutsche Staatskonzeption verfolgt. Die Diskussion, ob angesichts der Entwicklung in der Schweiz und den USA 1848 nicht doch ein deutscher Bundesstaat möglich gewesen sei, bleibt kontrovers. 


\section{Sektion: Die deutsche Nation von außen}

\section{Michael North: Die deutsche Nation und der Norden - Wirtschaftsraum und ethisch-kulturelle Grenzen}

Die Identität der deutschen Elite im Baltikum, die sich in der Gelehrtenrepublik verortet habe, steht im Zentrum dieser Diskussion. Mit seinen geographischen und historischen Arbeiten sei dieser Personenkreis im Reich auf große Resonanz gestoßen. Gefragt wird, ob es im Baltikum sich überlagernde oder gemeinsame kulturelle Räume dieser deutschen Bevölkerungsschicht und der einheimischen Oberschichten gegeben habe. Festzustellen sei, daß man in den von Adligen und Bürgern frequentierten Klubs beispielsweise über die Aufnahme russischer Militärs diskutiert habe. Eine Überlagerung von Identitäten zeige insbesondere der kurländische Adel, den seine deutsche Selbstzuschreibung wie seine Bindung an den russischen Hof prägten. Mit dem Ende der Dominanz des deutschen Adels am russischen Hof Mitte des 18. Jahrhunderts sei die Berufung auf die deutsche Identität erschwert worden, so daß fortan ein stärkerer Bezug auf eine kurländische Identität zu beobachten sei. In Reval könne zudem ein kultureller Assimilationsprozeß festgestellt werden: Esten, Letten und Deutsche hätten gemeinsame kulturelle Einrichtungen besessen. Die Ambivalenz des Identitätskonzeptes zeige aber Herders Postulat, Humanität erfordere Einsprachigkeit. Die deutschsprachigen Autoren hätten sich auch deswegen im Baltikum vielfach als isoliert erlebt. Aus Enttäuschung über ihre Lage, ihren „baltischen Frust“, seien auch die Lebensbedingungen der lettischen und livländischen Bevölkerung und die Leibeigenschaft in ihren Blick geraten. Mit ihren Publikationen hätten die deutschsprachigen Autoren wesentlich zur Abschaffung von Mißständen und etwa auch zur Verbesserung des Schulwesens beigetragen.

\section{Thomas Maissen: Die Eidgenossen und die deutsche Nation}

Wie läßt sich das Verhältnis zwischen der Schweiz und dem Reich charakterisieren? Die Schweiz habe jedenfalls verhältnismäßig lange an ihrer Reichszugehörigkeit festgehalten, denn diese begründete die Legitimation der kantonalen Herrschaftsrechte. Freilich hätten die Schweizer die habsburgische Position zurückgewiesen, wonach es sich bei der Schweiz um okkupierte habsburgische Gebiete handele.

An der „gestalteten Verdichtung“ des Reiches in der ersten Hälfte des 16. Jahrhunderts habe sich die Eidgenossenschaft jedoch nicht mehr beteiligt: Schweizer Autoren argumentierten, man sei im „Vor-Wormser Reich“ verblieben. Den Landfrieden könnten sie selbst besser und finanziell günstiger sichern als dies durch die Wormser Beschlüsse von 1495 möglich sei. Kantone mit besonders langen Grenzen zu deutschen Gebieten hätten allerdings ein stärkeres Interesse am Austausch mit dem Reich besessen, ähnlich wie die westlichen Gebiete mit ihrem französischen Umfeld. Selbst in der zweiten Hälfte des 17. Jahrhunderts sei der 
publizistische Einbezug der Schweiz ins Reich noch möglich gewesen. Die diplomatische Lösung vom Reich habe sich nach 1648 angesichts der Expansionskriege Ludwigs XIV. schwierig gestaltet.

Vorgeschlagen wird, das Verhältnis zwischen der Schweiz und dem Reich mit dem Begriff der „Reichsverwandtschaft“ zu fassen. Dieser Begriff könne als fünfte Kategorie das Raster des (1.) abendländisch-universal gedachten Reichs, des (2.) Lehensverbandes, des (3.) politischen Systems „Altes Reich“, das auf die deutschen Lande konzentriert war, und des (4.) kleinteiligen Kernreichs in Schwaben, Franken und am Rhein ergänzen. Entsprechend gebe es dann um die Mitte des 17. Jahrhunderts gehende (Schweden) und kommende Reichsverwandte (im Osten). Gegen den Begriff spreche aber, daß er - anders als die anderen vier Ebenen - keine konkreten Verpflichtungen impliziere.

Festgehalten wird, daß die Differenzerfahrung nicht unbedingt auf der Linie Schweizer - Deutsche verlaufe, denn sie sei vor allem durch Konfliktsituationen zwischen Schweizern und Schwaben entstanden. Die im 15./16. Jahrhundert aufkommende helvetische Identität lasse sich somit auch als ein Teil der deutschen Reichsidentität fassen. Dies gelte auch für die ästhetischen Konzeptionen der Schriftsteller. Seit dem frühen 16. Jahrhundert werde die Schweiz nicht mehr nur über militärische Helden definiert, sondern zunehmend auch kulturell. Der Bezug auf Tell sei allerdings von den Schweizer Obrigkeiten nicht gern gesehen worden, denn als Meuchelmörder habe er die Legitimation von Herrschaft gestört.

\section{Hans-Jürgen Bömelburg: Polen und die deutsche Nation}

Die Diskussion konzentriert sich auf die Wahrnehmung der deutschen Herrschaft im frühneuzeitlichen Polen. In der Publizistik, besonders während der Interregna, sei sie im Unterschied zur milden Herrschaft im polnischen Staatswesen als „tyrannisch“ dargestellt worden. Diese Beschreibung stehe im offensichtlichen Gegensatz zum deutschen Selbstbild. Die polnische Argumentation beruhe auf den Erfahrungen der habsburgischen Thronkandidaturen - beispielsweise habe diejenige Erzherzog Maximilians III. zum Bürgerkrieg geführt. Die sächsisch-polnische Union des 18. Jahrhunderts widerspreche freilich diesen Vorbehalten. Wenn der Deutsche in der polnischen Wahrnehmung gleichwohl der Fremde schlechthin geblieben sei, so beruhe dies auch auf den Berichten polnischer Adliger über ihre Besuche an deutschen Höfen, deren Soziabilität und Zeremoniell als ausgrenzend und nicht-partizipatorisch wahrgenommen worden seien.

Die deutschsprachigen Eliten in Polen wie in Danzig hätten ihre Sprache zwar als Distinktionskriterium genutzt, nicht aber mit einer Sprachnation argumentiert. Und während die frühe humanistische Literatur mit Tuiskon als Stammvater der Deutschen und der Polen eine Gemeinsamkeit kenne, sei dieses Motiv nach 1520/1530 kaum mehr nachzuweisen. Der Vergleich zwischen den Adelsrepubliken Polen und Venedig, ein Standart in der europäischen Publizistik, finde sich zwar in der Danziger Diskussion, sei von polnischer Seite aber nur schwach rezipiert worden. Maßgeblich hierfür sei wohl die Mitte des 17. Jahrhunderts erfolgte 
und ein Jahrhundert andauernde Abkoppelung der polnisch-litauischen Diskurse von den gesamteuropäischen. Erstaunlicherweise spielten aber auch die Nationalheiligen für die frühneuzeitliche Nationskonstruktion in Polen kaum eine Rolle. In Schlesien habe es allerdings wegen der Grenzlage ein recht ausgeprägtes Reichsbewußtsein gegeben. Die Ausrichtung von Kunst und Architektur an Böhmen und Süddeutschland nach 1648 sei dafür ein wichtiger Gradmesser. Die Protestanten hätten zudem den Schutz des Heiligen Römischen Reiches gesucht. Von polnischer Seite sei Schlesien aber zur Polonia gezählt worden.

\section{Martin Wrede: Frankreich, das Reich und die deutsche Nation}

Die Diskussion betont die Zäsur von 1648 für die französische Sicht auf das Reich. Die dortigen Autoren hätten danach über präzisere Kenntnisse verfügt, obwohl ihr Blick auf das Reich von den Rechtsgrundlagen ausgegangen und damit statisch geblieben sei. So habe die französische Diplomatie die Habsburger bis ins frühe 18. Jahrhundert in einem Gegensatz zum Reich gesehen. Aufgrund dieser Fehlperzeption seien keine dauerhafteren Erfolge mit der antikaiserlichen Politik zu erzielen gewesen. Die Idee, das Reich gegen den Kaiser zu organisieren, finde sich schon im Denken des Duc de Rohan in den 1630er Jahren. Dabei zeigten sich Kontinuitätslinien zum Agieren Heinrichs II. während des Fürstenaufstandes. Die veränderte Rolle Österreichs im 18. Jahrhundert hätte ebensowenig wie das Renversement des alliances entscheidenden Einfluß auf die großen Linien der französischen Außenpolitik gehabt. Als Glacis sei das Reich gleichwohl ein zentrales Interessengebiet der französischen Großmachtpolitik im 18. Jahrhundert geblieben - Napoleon könne insofern als Fortsetzer dieser Politik gelten. Den Titel eines Garanten der Reichsverfassung habe man in Frankreich als „größten Schmuck" des Königs gefeiert, der das Reich im Gleichgewicht halte. Wenn in französischen Buchtiteln der Begriff „Empire“ für das Reich verwendet worden sei, sei dies aus pragmatischen Gründen geschehen: Im Laufe des 18. Jahrhunderts finde sich jedoch „Allemagne“ häufiger, so daß das Reich aus französischer Sicht gleichsam deutscher werde. Karl den Großen hätten die frühneuzeitlichen französischen Autoren durchaus als Germanen dargestellt. Ihre Schriften seien auch in Deutschland rezipiert und Rezensionen zu wichtigeren Werken wie denjenigen von Voltaire oder Chrétien Frédéric Pfeffel unter anderem in den „Göttingischen Gelehrten Anzeigen" erschienen.

\section{Sektion: Distinkt oder übergreifend?}

\section{Horst Carl: Europäische Adelsgesellschaft und deutsche Nation}

Der „europäische Adel“ steht als Begriff und soziales Phänomen im Zentrum der Debatte. Unterschieden werden müsse die diskursive Ebene, die in der Frühen Neuzeit keine Vorstellung von einem europäischen Adel besitze, und der For- 
schungstopos „europäischer Adel“. Für die Existenz eines europäischen Adels seit dem Mittelalter sprächen insbesondere die dynastischen Verbindungen, der europäische Interaktionsraum und die adligen Militärkarrieren. Auch bei Cyriacus Spangenberg, dem Autor des wichtigsten Adelstraktats und Vertreter des Tugendadels, hätten nationale Argumente praktisch keine Rolle gespielt. Er gruppiere landschaftlich, habe aber im Dienst des reichsfreien Niederadels geschrieben, der stark regional verankert gewesen sei (Territorialadel). Doch zeigten sich auch hier übergreifende Erfahrungsräume, wenn Niederadlige Ritterakademien besucht oder die Grand tour absolviert hätten. Die Höfe spielten jedenfalls für den Kulturtransfer und eine supranationale Sozialisation eine wichtige Rolle. Kritik sei daran erst im 18. Jahrhundert vor allem aus merkantilen Gründen aufgekommen. Offen bleibt die Frage, ob Vorstellungen der „nationalen Ehre“ mit der Adelsehre korrelieren. Der in den Völkertafeln auftauchende Begriff „teutsche Treue“ sei jedenfalls nicht spezifisch auf den Adel bezogen worden. Zu den distinkten Elementen des deutschen Reichsadels zähle zudem seine Stiftsfähigkeit.

\section{Joachim Whaley: Kulturelle Toleranz - die deutsche Nation im europäischen Vergleich}

Inwieweit verändert sich die intellektuelle Konstruktion der deutschen Identität am Ende des 18. Jahrhunderts? Diese Frage dominiert die Aussprache. Mit dem historisierenden Denken seien nationalkulturelle „Blütezeiten“ denkbar geworden und für die damalige deutsche Nation beansprucht worden. Daraus sei aber kein Überlegenheitsbewußtsein abzuleiten, denn zeitgenössisch werde eher das Zeitalter der Menschheit postuliert und die Ära der Nation für beendet erklärt. Diskutiert werden anschließend die Quellen, die nationales Denken beinhalten: Sei dieses in Egodokumenten deshalb oft nicht greifbar, weil es sich um eine kulturelle Selbstverständlichkeit handele? In welchen Kontexten hätte es von den Zeitgenossen notwendigerweise thematisiert und verschriftlicht werden müssen? Das gedruckte Deutsch, das jeder verstanden habe, sei - wie etwa die Reichstagsakten zeigten - die „Amtssprache“ des Reiches gewesen. Thematisiert werden abschließend Stationen der Mobilisierung des Nationalgefühls in Europa seit dem Hundertjährigen Krieg sowie die Unterschiede in den verfassungsgeschichtlichen Strukturen Englands und Frankreichs.

\section{Siegrid Westphal: Franengelehrsamkeit im Wettstreit der Nationen am Beispiel der Frauenzimmerlexika}

Die Diskussion unterstreicht die große Bedeutung der Nation als Identifikationsangebot für beide Geschlechter. Die Gelehrsamkeit sei für die frühneuzeitliche deutsche Nation, die sich als Gelehrtenrepublik definiert habe, besonders wichtig gewesen. Weibliche Gelehrsamkeit zeige sich in einer breiten Palette anerkannter Fähigkeiten, zu denen auch musikalische gehörten, während die Naturwissenschaften als für Frauen nicht geeignet erschienen. Verwiesen wird auf den seman- 
tischen Wandel, den der Begriff der Gelehrsamkeit im 17. und 18. Jahrhundert durchlaufe. Die Frage sei, ob die Frauenzimmerlexika den Stellenwert einer Gattung der ernstzunehmenden Gelehrsamkeit erreicht hätten, obwohl sie nicht der Historia Literaria zugehörig gewesen seien. Den Zeitgenossen sei freilich deren mögliche Fiktionalität durchaus bewußt gewesen und letztlich könne auch eine Zusammenstellung von Verhaltensidealen und des halbwegs Seriösen („Buntschriftstellerei“) dem politischen Mythos, der Erhöhung der eigenen Nation und der Schaffung von Heldinnen einer nationalen Erinnerungskultur dienen. Im Laufe des 18. Jahrhunderts sei der Unterhaltungsaspekt stärker geworden. Wenn die Gattung der Frauenzimmerlexika auch um 1800 zunächst ihr Ende gefunden habe, lebe doch die Idee von vorbildlichen Frauen als Identifikationsfiguren für die weibliche Welt und die Nation in anderen Medien fort.

\section{Luise Schorn-Schütte: Politica christiana. Eine christliche oder konfessionelle Grundordnung für die deutsche Nation?}

Die Diskussion thematisiert den konfessionellen oder christlichen Charakter der Politica christiana: Seit dem 17. Jahrhundert sei sie überkonfessionell eine christliche Grundordnung für die deutsche Nation gewesen. Mit ihr könne somit eine politische Ideengeschichte der Frühen Neuzeit geschrieben werden. Gefragt wird, ob die Politica christiana als Theorie oder Subtheorie bzw. als Legitimitätsmodell zu verstehen sei, das flexibel und anlaßbezogen vor allem an die Verhältnisse der mittleren Territorialstaaten des Reiches habe angepaßt werden können. Festgestellt wird, daß es sich in dem Sinne um eine Theorie handele, daß ihre Beschreibung von Herrschaft mit dem Anspruch auf Umsetzung erfolge. Den Autoren sei die Verortung der Souveränität wichtig gewesen, deshalb könne nicht von einer reinen Verwaltungsanweisung unter dem Leitbegriff der administratio statt der gubernatio imperii gesprochen werden. Bis zum Ende des 17. Jahrhunderts sei daher von einer handlungsorientierten Herrschaftslehre auszugehen. Das Konzept der Politica christiana finde sich auch bei Theodor von Reinkingk. Seine Schrift „Biblische Policey“ (1653) verstehe sich vor dem Hintergrund der zeitgenössischen Ständekonflikte, insbesondere des hessischen, in dem der Fürst stark mit der necessitas argumentiert habe. Reinkingk betone darüber hinaus die Handlungskompetenz der Kurfürsten und die Rolle des Kaisers als mentor ecclesiae.

Die Aussprache thematisiert weiterhin verschiedene Legitimationsformen von Widerstand, die katholisch geprägte Politica christiana, der der Fürst als Vertreter Gottes gilt und die den Amtsträgern das Notwehrrecht und der Geistlichkeit eine Mahnfunktion zuweist, sowie säkulare Modelle zur Begründung von Widerstand wie die juristische Figur der leges fundamentales und das Verständnis des Kaisers als limitierter Monarch. Die zeitgenössische Diskussion um den Prager Frieden zeige die Bedrohungswahrnehmung dieser Zeit. Festgehalten wird, daß der patria-Begriff in der Politica christiana systematisch angelegt sei. Es gebe auch ein christliches Naturrechtsmodell, das die lutherische Debatte über patria und Widerstand kenne. 


\section{Sektion: Nationalkulturelle Zuschreibungen}

\section{Daniel Fulda: Das Konzept der Nation in der deutschen Literaturgeschichts- schreibung des 18. Jabrbunderts}

Diskutiert werden Gottscheds Literaturprogramm, seine soziale Einbindung und seine Wirkung auf die deutschsprachige Literatur. Er habe Vertreter anderer Kulturmodelle wie des schlesischen Spätbarocks oder der Hamburger Literaturszene marginalisiert, indem er seinen breiten Mitarbeiterkreis, zu dem auch seine Frau zu zählen sei, und ein dichtes Korrespondentennetz in ganz Deutschland optimal genutzt habe. Seit Mitte der 1740er Jahre sei Gottscheds Einfluß jedoch rückläufig, zumal er sich beispielsweise nicht mehr gegen die Schweizer Autoren wie Johann Jakob Bodmer und Johann Jakob Breitinger habe durchsetzen können. Dieser Literaturstreit sei aber weniger als Kampf um die Deutungshoheit im deutschen Sprachraum, sondern als Bewußtwerden und Formulieren von Differenzen zu verstehen. Allgemein werde den von den Schweizer Literaten vertretenen Leitwerten - Freiheit, politisches Engagement, Abgrenzung vom Höfischen und Orientierung am Republikanischen - von deutscher Seite eine apolitische Haltung entgegen gesetzt. Dabei zeige sich aber eine Asymmetrie der Sichtweisen: Während von den Schweizern selbst als Besonderheit ihrer Literatur das sich in ihr äußernde besondere Freiheitsbewußtsein betont worden sei, sei dies von der deutschen Seite kaum gewürdigt worden.

Bei Gottsched ließen sich freilich dezidierte Publikationsstrategien nachweisen: Anders als beispielsweise Friedrich Nicolai habe er das Konfessionelle zurückgedrängt, um in katholischen Territorien wirken zu können. Diese Überlegungen reichten bis zu den Akademieplänen in Wien. Auch sei er mit den Grenzen der Sprachnation pragmatisch umgegangen und habe die Niederländer als geborene Deutsche bezeichnet, um ihre Erfolge im Handel vereinnahmen zu können. Die Vorstellung aber, daß die Literatur als Vorstufe einer politischen Einheit der Nation diene, sei dem 18. Jahrhundert fremd gewesen. Erst die Literaturgeschichte des 19. Jahrhunderts habe die Literatur als erste Stufe für die Verheißung und Entstehung der Nationalstaaten verstanden.

Angesprochen wird auch das Weiterwirken des Humanismus und seiner Topoi. Allerdings hätten die konfessionellen Frontstellungen die Kontinuität vom Gelehrtenhumanismus bis zum 18. Jahrhundert verhindert, obwohl dieser in der zweiten Hälfte des 17. Jahrhunderts im Kontext antifranzösischer Haltungen und der Ablehnung des Höfischen einen neuerlichen Aufschwung genommen habe. $\mathrm{Daß}$ die konfessionelle Problematik nicht überschätzt werden dürfe, zeige aber der Blick auf den ostmitteleuropäischen Späthumanismus, der nicht vergleichbar konfessionell aufgeladen gewesen sei. 


\section{Klaus Pietschmann: Nationale Identitätskonstruktion im deutschsprachigen Musiktheater des ausgehenden 18. Jabrbunderts}

Das deutschsprachige Musiktheater des späten 18. Jahrhunderts stelle sich teilweise in den Dienst der nationalpatriotischen Idee. Für die Akzeptanz dieser Werke seien jedoch nicht nur die patriotischen Sujets und Motive wichtig gewesen, sondern ob sie ästhetisch zu überzeugen und dadurch das Publikum anzusprechen vermocht hätten. Im nationalpädagogischen Ansatz der Oper „Günther von Schwarzburg“ (1777), in der Erweiterung des Freiheitsprogramms von den Fürsten auf die Völker wie in der Sakralisierung der Nation, zeigten sich Parallelen zu Friedrich Carl von Mosers „Vom deutschen Nationalgeist“ (1765). Ebenso biete sich ein Vergleich mit dem Singspiel „Alceste“ an, das als erste Oper mit deutschem Libretto gelte, wenn es auch noch am französischen Vorbild ausgerichtet gewesen sei. Sein Erfolg beruhe auf dem Wielandschen Libretto; die Musik Anton Schweitzers sei bereits zeitgenössisch als defizitär eingeschätzt worden, was langfristig zum Scheitern des Stückes geführt habe.

\section{Meinrad von Engelberg: "Deutscher Barock“ oder „Barock in Deutschland“- nur ein Streit um Worte?}

Die Diskussion dreht sich um die Bedeutung der Begriffe „deutsch“ und „Deutschland“ für das Barockzeitalter und die sich daraus ergebenden Eingrenzungsprobleme für eine neue Architekturgeschichte dieser Zeit. Zu unterscheiden sei zwischen dem Terminus „deutsch“ auf der einen und „national“ bzw. „Reich“ auf der anderen Seite. Da das Publikum einer solchen Architekturgeschichte von den heutigen Begriffen „deutsch“ und „Deutschland“ und den heutigen Staatsgrenzen ausgehe, seien diese auch zugrunde zu legen. Die Rede vom „Barock in Deutschland" erlaube daher nicht den Einschluß des böhmischen und österreichischen Barock. Beim Begriff „deutsche Kultur" gehe es im Kern um Erinnerungskultur. Die Frage laute, was in langer Tradition zu ihr gehöre - auf jeden Fall doch wohl Schlesien mit seinen Friedenskirchen. Es sei aber nach Eigenarten und möglichen nationalen Merkmalen von Kunst zu fragen, vergleichbar dem Ansatz Heinz Schlaffers in seiner „Kurzen Geschichte der deutschen Literatur" (2002). Dabei müsse zwischen durchgängigen und nicht durchgängigen Merkmalen unterschieden werden. Überlegt wird, ob ein konstituierendes Element des deutschen Barock in seiner besonderen Offenheit gegenüber Einflüssen von außen bestehe und ob insofern von einer "Eigenart in der Uneigenart" der deutschen Kunst gesprochen werden könne. Festgehalten wird, daß stets das Thematisieren und Offenlegen des gewählten Ansatzes notwendig sei. Andere Zugriffe wie Thomas DaCosta Kaufmanns Formel von der „Kunst in Mitteleuropa“ seien ebenso problembehaftet wie die Konzeption „nationaler“ Kunstgeschichten. 